\title{
Kupferwerkstoffe im Sekundärteil eines linearen Asynchronmotors - Betrachtung der mechanischen Eigenschaften
}

\author{
Ivo Maximow*, Tobias Schöneck, Sebastian Weise, Markus Golder \\ Professur Förder- und Materialflusstechnik, Technische Universität Chemnitz, Germany \\ * Korrespondenz: ivo.maximow@mb.tu-chemnitz.de \\ Erhalten am 9. Oktober 2020; Angenommen am 4. November 2020; Online zugänglich seit 7. Dezember 2020 \\ (C) 2020 by I. Maximow u.a. Dies ist ein Open-Access-Artikel, der unter der Creative Commons Attribution License \\ (CC-BY 4.0) verbreitet wird, die die uneingeschränkte Nutzung, Verbreitung und Vervielfältigung in jedem Medium \\ erlaubt, vorausgesetzt, das Originalwerk wird ordnungsgemäß zitiert.
}

\begin{abstract}
In dem Forschungsprojekt wird ein berührungsloses Direktantriebssystem für hochdynamische Bandförderer entwickelt. Die Förderkraft wird nach dem Prinzip der linearen Asynchronmaschine in den Gurt eingeleitet. Zur Umsetzung des Antriebsprinzips muss der Gurt flächig eine Kupferstruktur, die den Sekundärteil der Asynchronmaschine darstellt, beinhalten. Die Herausforderungen bei der Auslegung dieser Struktur bestehen in der Berücksichtigung der elektrischen Anforderungen zur Umsetzung des Linearmotors einerseits und anderseits in der Berücksichtigung der mechanischen Anforderungen die während des Betriebs des Förderers auftreten. Im weiteren Projektverlauf wird das Antriebssystem um Condition-Monitoring-Funktionalitäten erweitert: Anhand der Statorströme wird die elektrodynamische Rückwirkung der Kupferstruktur im Gurt ausgewertet. Die gewonnenen Daten ermöglichen einen Rückschluss auf den Zustand der Kupferstruktur und gestatten somit eine Beurteilung des Verschleißzustandes des Motors und des Gurts. Ferner lässt sich der Bewegungszustand des Bandförderers bestimmen. Aus wirtschaftlichen Gründen soll sich der resultierende Bandförderer mit neuartigem Antriebssystem durch kleine Umlenkradien, den Verzicht auf zusätzliche Zugträger sowie die Erreichung eines bestmöglichen motorischen Wirkungsgrades auszeichnen. Für diese Forschungsziele ist die Kupferstruktur, welche in den Gurt integriert wird, von herausragender Bedeutung. In diesem Beitrag wird das Ergebnis der Betrachtung der Kupferwerkstoffe dargestellt.
\end{abstract}

KEYWORDS Bandförderer, Direktantrieb, Asynchronmaschine, Kupferwerkstoff, Biegebeanspruchung

\section{Einleitung}

Bandförderer zeichnen sich durch ihre universellen Einsatzmöglichkeiten aus. Es können verschiedenste Stück- oder Schüttgüter gefördert werden. Zudem erreichen sie höchste Fördergutdurchsätze. Die Antriebskraft wird herkömmlich über eine reibschlüssige Verbindung eingeleitet. Aus diesem reibschlüssigen Antrieb geht die Anforderung hervor, dass das Zugmittel gespannt werden muss, um eine Kraft übertragen zu können. [1] 
Daraus resultieren die folgenden Nachteile:

- Es sind zusätzliche Spanneinrichtungen notwendig.

- Bei reibschlüssiger Kraftübertragung ist grundsätzlich der Einsatz bei Anwendungen mit Umgebungsmedien, die den Reibkoeffizienten zwischen Trommel und Zugmittel beeinflussen, problematisch.

- Prinzipiell sind eine ideale Ausrichtung von Trommeln sowie eine ideale Fertigung von Zugmitteln nicht möglich. Unter Spannung neigen Gurte daher immer zum Schieflauf und müssen über Seitenführungseinrichtungen verfügen. Zudem sind sie nur eingeschränkt reversierend betreibbar.

- Bei der Übertragung des Drehmomentes zwischen Trommel und Zugmittel treten mikroskopische Gleitbewegungen im Nutzbogen der Umschlingung zwischen den beiden Komponenten auf. Dieser Dehnungsschlupf ist auf die hohe Elastizität des Zugmittels zurückzuführen und bewirkt Abrieb am Fördersystem.

Trotz der genannten Nachteile ist der Gurt als Zugmittel für einige Förderanwendungen aufgrund der kontinuierlichen und geschlossenen Oberfläche sowie dem hohen erreichbaren Durchsatz alternativlos. Darüber hinaus sind mit Gurtförderern sehr kleine Übergabestellen erzielbar. Die folgenden Anwendungsbeispiele des Gurtförderers sind dabei besonders von den Systemnachteilen einer reibschlüssigen Krafteinleitung betroffen.

- In der Lebensmittelindustrie werden hohe Anforderungen an die Reinigbarkeit und die hygienischen Eigenschaften gestellt. Hierbei ist die geschlossene Oberfläche ohne Toträume ein wichtiges Auswahlkriterium für den Gurt als Zugmittel. Gleichzeitig sind die Umgebungsbedingungen aufgrund von Nässe, Ölen, Fetten oder Seifen für eine reibschlüssige Kraftübertragung nachteilig.

- In der Lebensmittelindustrie ist für bestimmte Anwendungen der Abrieb infolge des Dehnungsschlupfs problematisch und muss durch Reinigungseinrichtungen entfernt werden.

- In der Pharma- und Kosmetikindustrie herrschen ähnliche Anforderungen wie in der bereits aufgeführten Lebensmittelindustrie. Hierbei ist Pulver als besondere Umgebungsbedingung zu nennen, welche den Reibschluss beeinflusst.

- In Recyclinganlagen führt die ungleichmäßige Beladung und starke Verschmutzung zu Problemen. Da die Fördergüter alle erdenklichen Strukturen, Größen und Oberflächen besitzen, kann nur der Gurtförderer aufgrund seiner breiten Einsatzmöglichkeiten verwendet werden.

Im Forschungsprojekt wird ein Antriebssystem für Bandförderer entwickelt, welches die genannten Nachteile des kraftschlüssigen Antriebs nicht aufweist. Nach dem Prinzip der Asynchronmaschine wird in ein Förderband, welches im Inneren flächig Kupfer enthält, eine Antriebskraft berührungslos eingeleitet. Hierbei beruht die Kraftentwicklung auf der Wechselwirkung zwischen induzierten elektrischen Wirbelströmen im Kupfer des Gurtes und dem magnetischen Wanderfeld des Motorstators, vgl. Abbildung 1. Das Kupfer stellt demnach das Sekundärteil einer linearen Asynchronmaschine dar. 


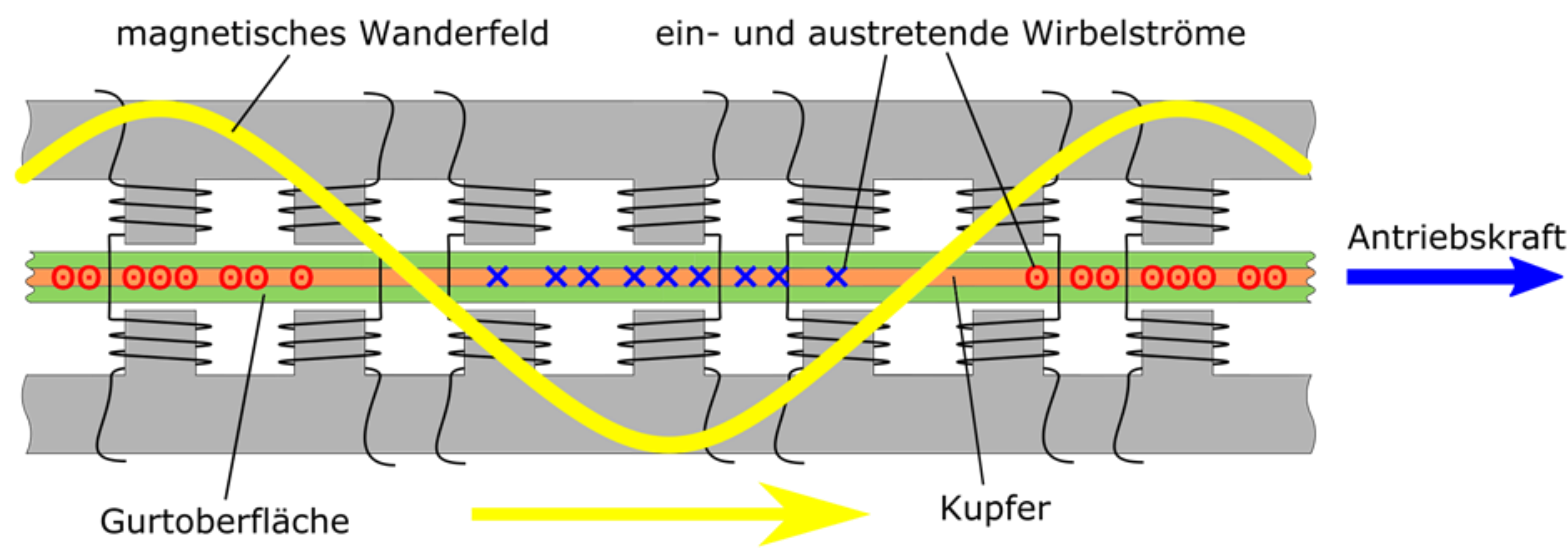

Abbildung 1: Skizze der umgesetzten linearen Asynchronmaschine für Bandförderer

Das beschriebene Antriebsprinzip wird ebenfalls zum Einprägen einer Kraft quer zur Förderrichtung angewendet. Damit ist die Umsetzung einer elektrisch regelbaren Geradführung möglich. Durch die Kombination von Antriebs- und Querkraft lässt sich ein hochdynamisches, reversierbares Antriebssystem für Bandförderer umsetzen, welches unabhängig von Reibpaarungen und Medieneinflüssen arbeitet. Da der Gurt Bestandteil des Antriebssystems ist, soll anhand der Rückwirkungen auf die Statorströme eine Diagnose des Gurtzustands und der Gurtbewegung umgesetzt werden.

Lineare Drehstrommaschinen wurden in den 70er und 80er Jahren intensiv erforscht. Zusammenfassende Werke mit Anwendungsbezug aus dieser Zeit sind [2] und [3]. Lineare Asynchronmaschinen als Direktantrieb für Bandförderer wurden damals für den Einsatz in großen Förderanlagen erforscht. So wurde in [4] die Möglichkeit des Einsatzes als Antrieb für Förderbänder in Braunkohletagebau untersucht. Die Patentanmeldungen [5] und [6] zeigen, dass auch Konzerne sich mit Linearmotoren für Bandförderer auseinandergesetzt haben. Dennoch hat sich die Technik nicht etablieren können. Dies ist unter anderem auf den Herstellungsaufwand und die Kosten für den Gurt zurückzuführen.

Im Gegensatz zu den beschriebenen Arbeiten ist das Antriebssystem, welches in diesem Projekt umgesetzt wird, für kleinere und sehr dynamische Bandförderer vorgesehen, sowie für Förderer, die empfindlich gegenüber Umgebungseinflüssen sind. Dabei soll der Bandförderer weiterhin seinen einfachen Aufbau beibehalten und möglichst günstig herzustellen sein. Hervorzuheben ist der Ansatz, eine regelbare Gurtführung und eine Diagnose des Bandförderers $\mathrm{zu}$ integrieren. Unter der aktuellen Forschung bezüglich Linearantriebtechnik für Förderanlagen sind [7] und [8] zu nennen. Diese wenden andere motorische Prinzipien an und haben einen grundsätzlich anderen Gurtaufbau. Das Hauptziel dieser Antriebssysteme besteht darin, entlang der gesamten Förderstrecke eine Antriebskraft einleiten zu können. 
In diesem Beitrag werden die Untersuchungen bezüglich möglicher Kupferwerkstoffe als Sekundärteil des Antriebssystems zur Integration in einen Gurt vorgestellt. Durch die Kombination von Motor und Zugmittel übertragen sich die Anforderungen des Bandförderers auch auf das Sekundärteil. Die folgenden Punkte leiten sich als Zielstellung für die Entwicklung des direkt angetriebenen Gurts ab:

- Um einen möglichst hohen motorischen Wirkungsgrad zu erreichen, muss das Sekundärteil eine hohe elektrische Leitfähigkeit aufweisen.

- Um kleine Übergabestellen zu realisieren, wird ein Gurtumlenkradius von $15 \mathrm{~mm}$ angestrebt.

- Die Zugkräfte im Gurt werden durch das Sekundärteil im Gurt übertragen. Dadurch kann auf zusätzliche Zugträger verzichtet und eine wirtschaftlichere Fertigung erreicht werden.

Die in diesem Projekt auftretenden Anforderungen an das Sekundärteil und dessen Werkstoff sind ungewöhnlich. Der Beitrag fasst die entstehenden Beanspruchungen zusammen und bewertet die Machbarkeit mit verfügbaren Werkstoffen und Halbzeugen.

\section{Werkstoffbetrachtung zur Integration des Sekundärteils in Bandförderer}

Die Anforderung an das Sekundärteil, eine hohe Leitfähigkeit aufzuweisen, schränkt die Auswahl der Werkstoffe stark ein. In Tabelle 1 sind die fünf elektrisch leitfähigsten Metalle mit Kennwerten angegeben.

Aus wirtschaftlichen Gründen ist der Einsatz von Silber, obwohl es die höchste elektrische Leitfähigkeit besitzt, und Gold ausgeschlossen. Eine Betrachtung von Aluminium zeigt, das neben der elektrischen Leitfähigkeit auch die Zugfestigkeit und Bruchdehnung gegenüber Kupfer geringer sind. Die mechanischen Eigenschaften von Aluminium können durch Legierung stark verbessert werden. Die elektrisch relevanten Legierungen in [11] übertreffen dennoch nicht Kupfer und weisen zudem noch geringere elektrische Leitfähigkeit als reines Aluminium auf [11]. Demzufolge wird für die weiteren Betrachtungen Kupfer als Material für das Sekundärteil ausgewählt.

Tabelle 1: Vergleich technisch reiner Metalle bezüglich elektrischer Eigenschaften $[9,10]$

\begin{tabular}{lll}
\hline Metall & $\begin{array}{l}\text { Relative elektrische Leit- } \\
\text { fähigkeit bezogen auf Kupfer } \\
\text { (Kupfer = 100) }\end{array}$ & $\begin{array}{l}\text { Temperaturkoeffizient des } \\
\text { elektrischen Widerstands } \\
\left(\mathbf{0 - 1 0 0 ^ { \circ }} \mathbf{C}\right) \text { in 10-3/K }\end{array}$ \\
\hline Silber & 106 & 4,1 \\
Kupfer & 100 & 4,3 \\
Gold & 72 & 3,9 \\
Aluminium & 62 & 4,2 \\
Magnesium & 39 & 4,2 \\
\hline
\end{tabular}




\subsection{Kupferwerkstoffe}

\section{Einteilung, Bezeichnungen und Normen}

Die vielfältigen Kupferwerkstoffe unterteilen sich prinzipiell in legiert und unlegiert. Letztere werden grundsätzlich in sauerstoffhaltig, sauerstofffrei und sauerstofffrei/desoxidiert unterschieden. Die Bezeichnungen der jeweiligen Werkstoffe variiert je nach Norm stark. In Europa ist ein Werkstoffnummernsystem [12] mit zusätzlicher Zustandsbezeichnung [13] genormt. Parallel dazu verwenden die europäischen Normen zusätzliche Werkstoffkurzzeichen, die aus der ISO 1190-1 entnommen sind. Im amerikanischen Raum gilt die Normung [14] mit eigenem Werkstoffnummernsystem und Kurzbezeichnungen.

Für das Forschungsprojekt ist vor allem Bandmaterial interessant, da es sich bedingt durch seine Form für die Integration in Gurte eignet. Für Bänder gilt die Norm [15], darin werden auch die chemischen Bestandteile definiert. Einen Überblick über alle Produkte und Zusammensetzungen von Kupfer und Kupferlegierungen wird in der Norm [16] gegeben.

\section{Einfluss von Legierungselementen und Verformungszustand}

Das Legieren von reinem Kupfer kann zu anderen und neuartigen Eigenschaften führen [10]. Für das Forschungsprojekt ist primär der Einfluss auf die elektrische Leitfähigkeit relevant. Diesbezüglich hat das Legieren immer einen negativen Effekt. In Abbildung 2(a) ist der Verlauf der elektrischen Leitfähigkeit unter Beimengung ausgewählter Elemente dargestellt [11]. Grundsätzlich ist der negative Legierungseinfluss bei Mischkristallbildung größer bei einem heterogenen Kristallgemisch [9].

Der Einfluss des Verformungszustands ist in Abbildung 2(b) dargestellt [11]. Eine Kaltumformung führt zu Erhöhung der Festigkeit bei Verringerung der Bruchdehnung. Diese
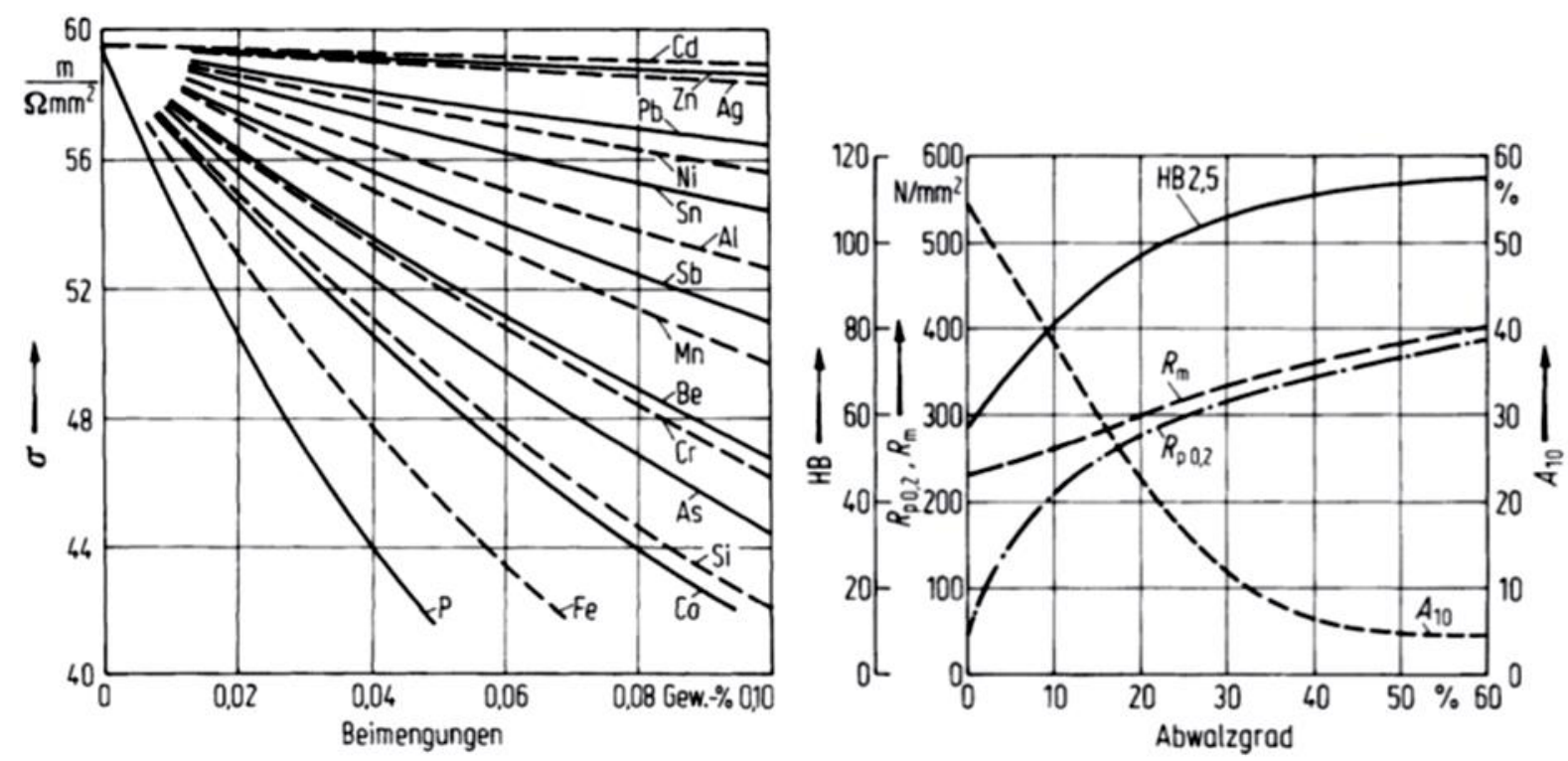

Abbildung 2: (a) Einfluss der Beimengung von Legierungselementen auf die elektrische Leitfähigkeit $\sigma$ von Kupfer [11]. (b) Einfluss des Verformungszustands auf die mechanischen Eigenschaften Härte HB, Streckgrenze $R_{p 0,2}$, Zugfestigkeit $\mathrm{R}_{\mathrm{m}}$ und Dehnung $\mathrm{A}_{10}$ von Kupfer (sauerstofffrei, nicht desoxidiert) [11]. 
Effekte können durch eine anschließende Wärmebehandlung wieder ausgeglichen werden [11]. Umfangreiche Kennwerte zu verschiedenen Verformungszuständen werden in [17] angegeben.

\section{Dauerfestigkeit}

Das kubisch-flächenzentrierte Metall Kupfer und die meisten seiner Legierungen weisen keine ausgeprägte Dauerschwingfestigkeit auf. Damit gehört es zum Wöhler-Kurventyp II [18]. Allerdings ist hierbei zu beachten, dass unlegiertes Kupfer in verschiedenen Zuständen bezüglich des Sauerstoffgehaltes vorliegt und auch der Verformungszustand die mechanischen Eigenschaften beeinflusst. In [19] wird eine Vielzahl von Dauerfestigkeitskurven von Kupferwerkstoffen mit unterschiedlichsten Zusammensetzungen und Verformungsgraden publiziert. Grundsätzlich lässt sich daraus ableiten, dass ein zunehmender Verformungsgrad einen positiven Einfluss auf die Dauerfestigkeit hat. Kaltgewalztes Kupfer mit einem geringen Phosphorgehalt von 0,01 bis 0,03\% zeigt beispielsweise ab ca. $10^{7}$ Zyklen ein nahezu dauerfestes Verhalten [9]. Eine Belastung von ca. $100 \mathrm{~N} / \mathrm{mm}^{2}$ wird dabei ertragen. Dass Phosphor die Festigkeitseigenschaften verbessert, wird zudem in [20] angeführt. Für weiches, nicht verfestigtes Kupfer wird in [9] ein Richtwert von $70 \mathrm{~N} / \mathrm{mm}^{2}$ für die Dauerschwingfestigkeit angegeben. Dabei wird nicht erläutert, ob der Sauerstoffgehalt diese Eigenschaft beeinflusst.

\section{Halbzeuge}

Grundsätzlich kommen für die Integration in den Gurt die Halbzeuge „Litze“ oder „Band“ in Frage, da diese, abhängig von ihren geometrischen Abmessungen, gute Biegeeigenschaften besitzen. Für die weiteren Untersuchungen innerhalb dieses Projekts soll Band verwendet werden, da bei diesem Halbzeug der Einfluss durch strukturbedingte Effekte ausgeschlossen werden kann. Die Beanspruchung von Litzen für Bandförderer kann mit der von laufenden Drahtseilen verglichen werden. Aufgrund der Struktur aus vielen Einzeldrähten, die sich zueinander verschieben können, treten unter anderem Biege-, Torsions-, sekundäre Zugund Biegespannungen auf. Umfangreiche theoretische Betrachtungen bezüglich der Belastung von Drahtseilen werden in [21] erläutert. Darüber hinaus hat der Aufbau der Litze und dessen Herstellungs- und Verarbeitungsprozess, beispielsweise das Walzen und Strecken, einen Einfluss auf die mechanischen Eigenschaften. Eine Berechnung der Belastung sowie die Reproduzierbarkeit von Versuchen sind dadurch wesentlich komplexer im Vergleich zu Bändern. Gegen die Verwendung von Litzenhalbzeug spricht des Weiteren, dass unter Biege- und Zugbeanspruchung mit dem Auftreten von Reibkorrosion zu rechnen ist. Näheres hierzu wird in [22] erläutert. Durch Korrosion wird neben den mechanischen Eigenschaften auch die elektrische Übertragung beeinflusst. Für das Wirkprinzip des Antriebssystems ist ein Stromfluss auch über den Oberflächenkontakt von Litze zu Litze notwendig. Dieser würde durch eine korrodierte Schicht negativ beeinflusst werden.

\subsection{Auftretende mechanische Belastungen im Förderer}

Nach jetzigem Stand wird davon ausgegangen, dass das Sekundärteil die Zugkräfte des Gurts überträgt. Die Gurtoberfläche wird aus einem Kunststoff bestehen, der eine deutlich geringere Steifigkeit als Kupfer aufweist. Demnach ist anzunehmen, dass das Kupferband 
die Lage der neutralen Faser des Gurts bestimmt. Nach derzeitigem Stand sollen Kupferbänder von 0,05 $\mathrm{mm}$ bis maximal 0,2 $\mathrm{mm}$ Dicke verwendet werden. Bei einem Umlenkradius von $15 \mathrm{~mm}$ kann, aufgrund der sehr geringen Stärke, von einer schwachen Krümmung des Bandes mit symmetrischer Lage der neutralen Faser ausgegangen werden [23]. In der folgenden Tabelle 2 sind die Dehnungen außen für verschiedene Kombinationen von Bandstärken und Umlenkradien angegeben. Eine Bewertung im Bezug zum Werkstoff erfolgt in den folgenden Abschnitten.

Tabelle 2: Banddehnung außen abhängig von Bandstärke und Umlenkradius

\begin{tabular}{lll}
\hline Bandstärke [mm] & Umlenkradius [mm] & Banddehnung [\%] \\
\hline 0,05 & 15 & 0.166 \\
& 30 & 0,083 \\
& 45 & 0,056 \\
\hline 0,2 & 15 & 0,662 \\
& 30 & 0,332 \\
& 45 & 0,222 \\
\hline
\end{tabular}

Da der Gurt aufgrund des Antriebsprinzips nicht vorgespannt werden muss, hängt die Zugkraftbelastung im Band ausschließlich von der Antriebskraft ab. Da das System für kleine dynamische Bandförderer vorgesehen ist, wird derzeit mit einer Antriebskraft von $200 \mathrm{~N}$ kalkuliert. Für eine Gurtbreite von $200 \mathrm{~mm}$ ergibt sich daraus für die geringste Bandstärke von 0,05 mm eine Zugspannung von $16 \mathrm{~N} / \mathrm{mm}^{2}$. Dieser Wert liegt deutlich unter der Streckgrenze, selbst für Werkstoffe ohne Verfestigung durch Verformung, vgl. Abbildung 2(b) und [17].

Die Hauptbeanspruchung für das Kupferband stellt die Dehnung bei der Umlenkung dar. Da Kupfer ein duktiler Werkstoff ohne ausgeprägte Streckgrenze ist, kann für das E-Modul nur ein Ersatzwert angegeben werden. Dieser, sowie die Bruchdehnung hängen allerdings stark von dem Verformungszustand ab. Ausführliche Angaben zu mechanischen Kennwerten sind in [17] und [19] publiziert. Um die Belastung durch Dehnung bei der Umlenkung besser einschätzen zu können, wurde im Rahmen dieses Projektes das Spannungs-DehnungsDiagramm des konkreten Kupferwerkstoffes aufgenommen. Die Ergebnisse sind in den folgenden Abschnitten dargestellt.

\subsection{Auswahl des Kupferwerkstoffes}

Aus den vorangegangenen Betrachtungen heraus wurde für das weitere Vorgehen ein nichtlegierter und mit Phosphor desoxidierter Kupferwerkstoff gewählt. Für die Untersuchungen wird ein nicht verformter „weicher“ und ein stark verformter „harter“ Werkstoff verglichen. Auch wenn die Vermutung naheliegt, dass aufgrund seiner besseren mechanischen Eigenschaften der harte Werkstoff besser geeignet ist, soll auch die weiche Variante untersucht werden, um den Einfluss des Verformungszustands für diese spezielle Anwendung zu definieren. Die gebräuchlichen Bezeichnungen des Werkstoffes lauten „CuPHC“ bzw. „CW020A“ mit den Zustandsbezeichnungen „R200“ für weich und „R370“ für hart $[12,13,16]$. Der R-Wert bezieht sich auf die Mindestzugfestigkeit des Werkstoffes, bei R200 beträgt diese mindestens $200 \mathrm{~N} / \mathrm{mm}^{2}$. In [9] wird Cu-PHC dem „OFXLP“ bzw. 
„C10300“ aus [14] gleichgesetzt, die Zusammensetzungen sind auch nahezu identisch $[16,17]$. Allerdings wird in [14] der Werkstoff als sauerstofffrei und nicht desoxidiert kategorisiert.

$\mathrm{Cu}-\mathrm{PHC}$ zeichnet sich durch eine hohe elektrische Leitfähigkeit von mindestens $57 \mathrm{~m} / \Omega \mathrm{mm}^{2}$ aus $[9,24]$. Ein desoxidierter Werkstoff eignet sich zudem sehr gut für spätere Bearbeitung mittels Schweißen oder Löten ohne dass Wasserstoffversprödung einsetzt [24]. Dies ist im Hinblick auf einen umlaufenden Gurt, der eine Endlosverbindung benötigt, ein wichtiger Aspekt im späteren Projektverlauf. Zudem ist Cu-PHC als Bandmaterial verfügbar.

Im Vergleich dazu haben legierte Kupferwerkstoffe den Nachteil, dass die elektrische Leitfähigkeit immer geringer gegenüber unlegiertem Kupfer ist. Zudem ist die Verfügbarkeit als Bandmaterial nicht immer gegeben bzw. nicht wirtschlich in der Beschaffung. Sollte ein Projekterfolg mit dem ausgewählten Werkstoff nicht möglich sein, wird die Verwendung eines legierten Werkstoffes neu bewertet.

\section{Versuche}

Alle hier aufgeführten Versuche wurden auf der Prüfmaschine „inspekt 10“ mit Schulterproben gemäß DIN EN ISO 6892 [25] durchgeführt.

\subsection{Spannungs-Dehnung-Verhalten}

In der Abbildung 3 sind zwei repräsentative Spannungs-Dehnungs-Verläufe von Cu-PHC der Zustände R370 und R200 dargestellt. Im Vergleich zu den Bruchdehnungen in der Literatur $[9,11,17]$ werden erheblich geringere Werte erreicht. Dies ist auf den sehr dünnen Rechteckquerschnitt der Zugproben zurückzuführen. Versuche mit Stangenproben sollten deutlich höhere Werte erreichen. Für das Projektvorhaben ist der Versuch mit dünner Rechteckgeometrie zu bevorzugen, da dies besser den Einsatz im Bandförderer abbildet.

Anhand der Diagramme wird ersichtlich, dass beide Materialzustände den für Kupfer typischen duktilen Dehnungsverlauf ohne ausgeprägte Streckgrenze besitzen. Das weichere Material R200 erreicht eine deutlich höhere Bruchdehnung. Für die Dauerfestigkeit in Bezug auf Biegebeanspruchung ist allerdings der elastische Bereich des Werkstoffes interessant. Eine genaue Definition ist aufgrund des fließenden Übergangs von elastischem zu plastischem Bereich schwierig. Dennoch wird anhand des Diagramms ersichtlich, dass der härtere Werkstoff R370 bei einer Dehnung von ca. 0,15-0,2\% den elastischen Bereich verlässt, während dies beim weicheren R200 schon bei 0,03-0,05\% geschieht.

\subsection{Zug-Schwell-Versuche}

Um das Dehnungsverhalten speziell im Übergang von elastischem zu plastischem Bereich $\mathrm{zu}$ untersuchen, wurden Zug-Schwell-Versuche durchgeführt. In Abbildung 4 sind die Diagramme für die Zustände R200 und R370 für 150 Lastzyklen dargestellt. 

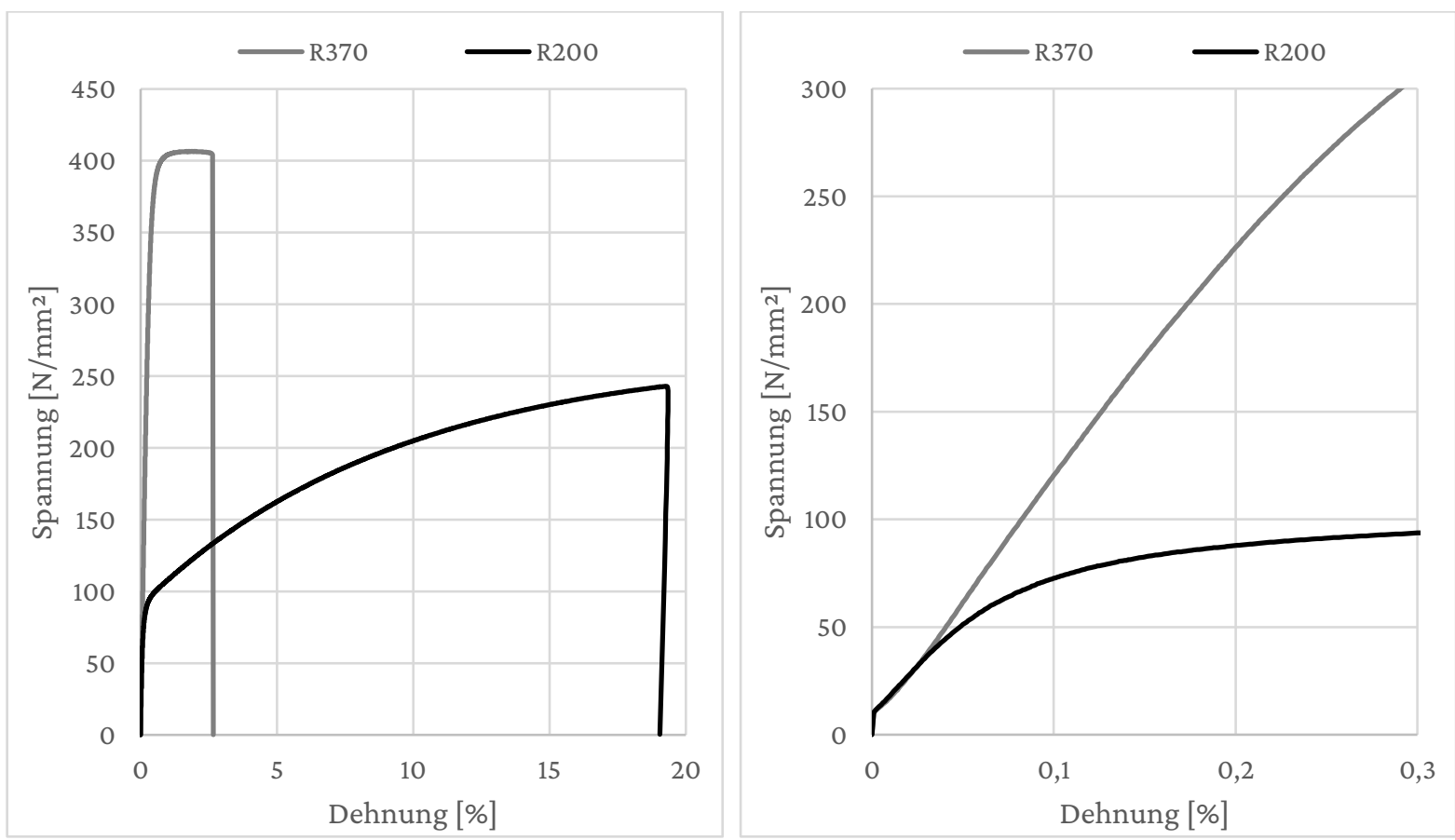

Abbildung 3: Spannungs-Dehnung-Diagramm für Cu-PHC Band der Stärke 0,2 mm in den Zuständen R370 und R200. (a) Ansicht über gesamten Dehnungsbereich, (b) Ansicht bis 0,3\% Dehnung
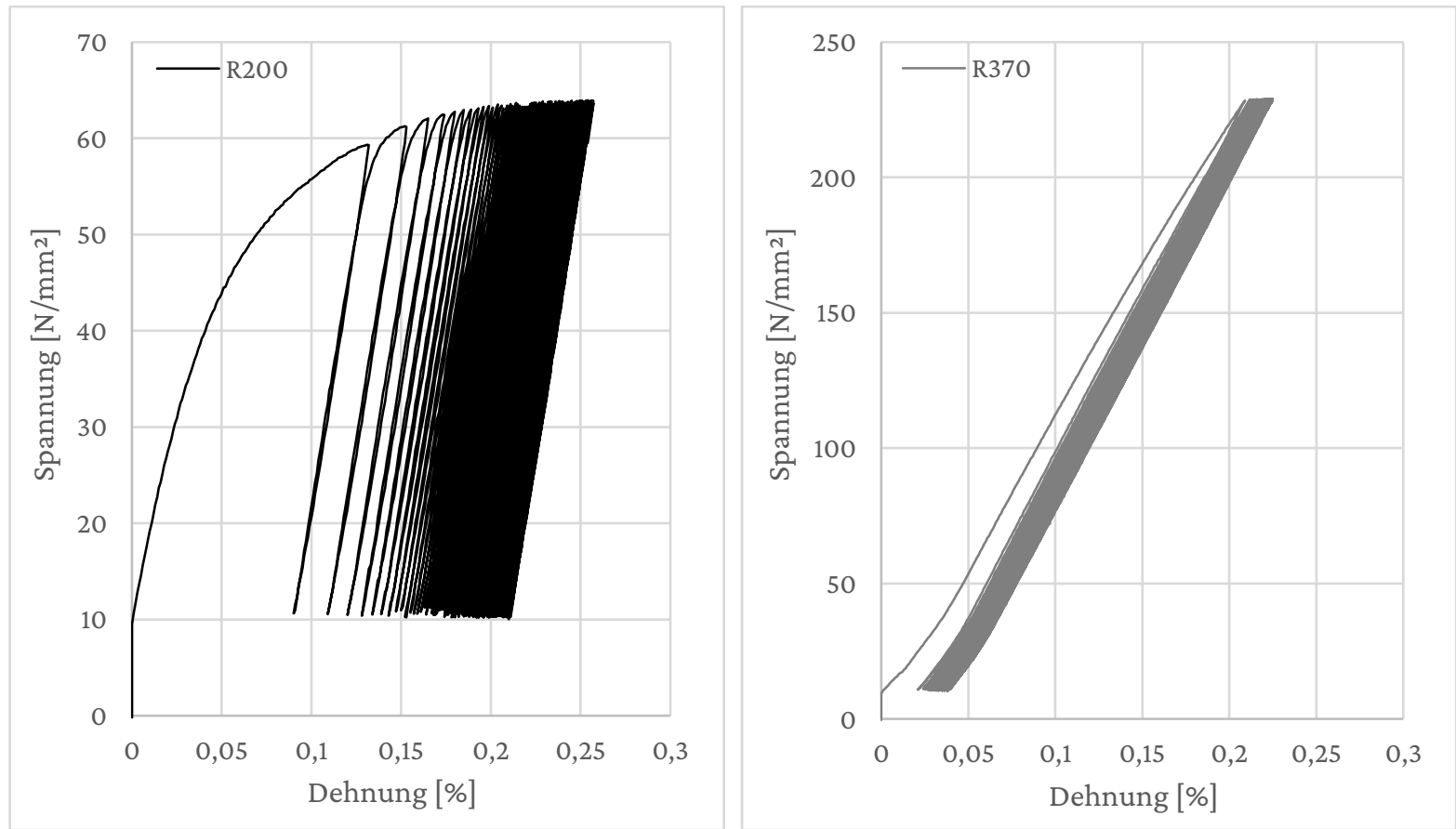

Abbildung 4: Verlauf des Spannungs-Dehnungsdiagramms von Cu-PHC Band der Stärke 0,2 mm über 150 Lastzyklen mit einer Belastung von (a) $64 \mathrm{~N} / \mathrm{mm}^{2}$ für R200 und (b) $230 \mathrm{~N} / \mathrm{mm}^{2}$ für R370.

Bei dem Material des Zustandes R200 steigerte sich in diesem Versuch die Dehnung bei Belastungsmaximum von $0,13 \%$ auf $0,257 \%$. Das entspricht einer Zunahme von $0,127 \%$. Dieser Wert fällt etwas hoch aus, da bei den ersten Zyklen die Last von $64 \mathrm{~N} / \mathrm{mm}^{2}$ nicht erreicht wurde. Dennoch ist über den gesamten Versuch festzustellen, dass sich das Material 
fortlaufend plastisch verformt. Mit zunehmender Zyklenanzahl sinkt die Rate des Dehnungszuwachses.

Bei dem Material des Zustandes R370 steigerte sich die Dehnung bei Belastungsmaximum von 0,212\% auf 0,225\%. Das entspricht einer Zunahme von 0,013\%. Ein Dehnungszuwachs ist nur zu Versuchsbeginn festzustellen.

Ein quantitativer Vergleich beider Materialien ist aufgrund der unterschiedlichen Belastungen schwierig. Dennoch lässt sich feststellen, dass das Material des Zustands R200 den Dehnungsbereich des analogen Versuchs mit Zustand R370 komplett durchlaufen hat und somit eindeutig schlechtere elastische Eigenschaften aufweist.

\section{Diskussion}

Anhand der durchgeführten Versuche lässt sich feststellen, dass Cu-PHC des Zustands R370 („hart“) gegenüber R200 („weich“) deutlich bessere elastische Eigenschaften besitzt und somit für den Einsatz mit mechanischer Biegebeanspruchung eher geeignet ist. Darüber hinaus erträgt es höhere Zugbelastungen. Das Material des Zustands R200 erreicht zwar eine wesentlich höhere Bruchdehnung, würde aber bei Gurtumlenkungen plastisch verformt werden und einem Dauerbetrieb nicht standhalten. Aus den Versuchen und Vorbetrachtungen lässt sich ableiten, dass Cu-PHC des Zustands R370 die Belastung im Bandförderer ertragen kann.

Um die Biegebeanspruchung unter realen Bedingungen mit überlagerter Zugbeanspruchung testen $\mathrm{zu}$ können, wird momentan ein Prüfstand entwickelt. Im Zusammenhang mit Biegewechselfähigkeit für Kupferwerkstoffe findet die Norm B470 [26] Anwendung. Allerdings ist diese für Kabel ausgelegt und beinhaltet Gegenbiegung, sodass eine Übertragung der Ergebnisse auf die hier beschriebene Anwendung nicht sinnvoll ist. Mit dem eigens für Bandförderer entwickelten Prüfstand sollen Dauerbiegeversuche bis zum Bruch durchgeführt werden. Anhand der gewonnenen Ergebnisse wird eine endgültige Aussage über die elastischen Eigenschaften der Kupferwerkstoffe sowie eine gezielte Dimensionierung für Bandförderer möglich sein.

\section{Förderhinweis}

Gefördert durch: Bundesministerium für Wirtschaft und Energie aufgrund eines Beschlusses des Deutschen Bundestages

\section{Quellen}

[1] Griemert, R., Römisch, P.: Fördertechnik. Auswahl und Berechnung von Elementen und Baugruppen, Seiten 217ff (Springer Vieweg, 2018)

[2] Luda, G.: Drehstrom-Asynchron-Linearantriebe. Grundlagen und praktische Anwendungen für industrielle Zwecke (Vogel-Verlag, 1981) 
[3] Budig, P.-K.: Drehstromlinearmotoren (VEB Verlag Technik, 1982)

[4] Noack, A.: Der Einsatz von Drehstromliniearmotoren für die Bandförderung in der Braunkohlenindustrie der DDR. Diss. (Technische Hochschule Karl-Marx-Stadt, 1981)

[5] Continental Gummi-Werke AG: Fördergurt. (Dt. Patentanmeldung 2336 294, 1975)

[6] Siemens AG: Fördereinrichtung mit elektrisch durch Wanderfeld angetriebenem endlosen Förderband (Dt. Patentanmeldung 2134 055, 1973)

[7] Kanus, M., Hoffmann, A., Overmeyer, L., Ponick, B.: Lineardirektantrieb für biegeschlaffe Transportbänder zur Reduzierung der Gurtzugkraft Tagungsband zum 15. Fachkolloquium der Wissenschaftlichen Gesellschaft für Technische Logistik e. V. (WGTL), Seiten 77-84 (2019)

[8] Radosavac, M., Overmeyer, L.: Entwicklung eines Lineardirektantriebs für Transportbänder, Logistics Journal (2016)

[9] Deutsches Kupfer-Institut e.V.: Kupfer in der Elektrotechnik - Kabel und Leitungen (Deutsches KupferInstitut Auskunfts- und Beratungsstelle für Verwendung von Kupfer und Kupferlegierungen, 2000)

[10] Fasching, G.: Werkstoffe für die Elektrotechnik. Mikrophysik, Struktur, Eigenschaften (Springer, 2005)

[11] Schuler, P., Vinaricky, E.: Aluminium und Aluminiumlegierungen, Kupfer und Kupferlegierungen. In: Elektrische Kontakte, Werkstoffe und Anwendungen. Grundlagen, Technologien, Prüfverfahren (Hrsg. Vinaricky, E.), Seiten 374-376, 377-431 (Springer Vieweg, 2016)

[12] DIN EN 1412:2017-01. Kupfer und Kupferlegierungen - Europäisches Werkstoffnummernsystem; Deutsche Fassung EN 1412:2016 (Beuth Verlag GmbH, 2017)

[13] DIN EN 1173:2008-08. Kupfer und Kupferlegierungen - Zustandsbezeichnungen; Deutsche Fassung EN 1173:2008 (Beuth Verlag GmbH, 2008)

[14] ASTM Standard: B224 - 16. Standard Classification of Coppers (ASTM International, 2008)

[15] DIN Deutsches Institut für Normung e. V.: DIN EN 1652:1998-03. Kupfer und Kupferlegierungen - Platten, Bleche, Bänder, Streifen und Ronden zur allgemeinen Verwendung; Deutsche Fassung EN 1652 : 1997 (Beuth Verlag GmbH, 1998)

[16] DIN CEN/TS 13388 (DIN SPEC 9700):2015-08. Kupfer und Kupferlegierungen - Übersicht über Zusammensetzungen und Produkte; Deutsche Fassung CEN/TS 13388:2015 (Beuth Verlag GmbH, 2015)

[17] Davis J. R., Hrsg.: ASM Specialty Handbook: Copper and Copper Alloys (ASM International, 2001)

[18] Läpple, V.: Einführung in die Festigkeitslehre. Lehr - und Übungsbuch. (Vieweg+Teubner Verlag, 2011)

[19] Reed, R. P., Mikesell, R. P.: „Low Temperature Mechanical Properties Of Copper and Selected Copper Alloys. A Compilation From the Literature. In: Monograph 101 (IMR, National Bureau of Standards, 1967)

[20] Deutsches Kupfer-Institut e.V.: Schweißen von Kupfer und Kupferlegierungen (Deutsches Kupfer-Institut Auskunfts- und Beratungsstelle für Verwendung von Kupfer und Kupferlegierungen, 2009)

[21] Feyrer, K., Wehking, K.-H.: FEYRER: Drahtseile. Bemessung, Betrieb, Sicherheit. (Springer Vieweg, 2018)

[22] Papailiou, K. O.: Bending of helically twisted cables under variable bending stiffness due to internal friction, tensile force and cable curvature (Eidgenössische Technische Hochschule Zürich, 1995)

[23] Niemann, G.: Maschinenelemente 1. Konstruktion und Berechnung von Verbindungen, Lagern, Wellen (Springer Vieweg, 2019)

[24] DIN EN 13602:2013-09. Kupfer und Kupferlegierungen - Gezogener Runddraht aus Kupfer zur Herstellung elektrischer Leiter; Deutsche Fassung EN 13602:2013 (Beuth Verlag GmbH, 2013)

[25] DIN EN ISO 6892-1:2020-06. Metallische Werkstoffe - Zugversuch - Teil 1: Prüfverfahren bei Raumtemperatur (ISO 6892-1:2019); Deutsche Fassung EN ISO 6892-1:2019 (Beuth Verlag GmbH, 2020)

[26] ASTM Standard: B470 - 02. Standard Specification for Bonded Copper Conductors for Use in Hookup Wires for Electronic Equipment (ASTM International, 2017) 